\title{
In-situ TEM Study of Mechanical Size Effects in TiC Strengthened Steels
}

\author{
S. Taniguchi ${ }^{1}$, R. Soler ${ }^{2}$, C . Kirchlechner ${ }^{2}$, C. Liebscher ${ }^{2}$, A. Taniyama ${ }^{3}$, and G. Dehm ${ }^{2}$ \\ 1. Advanced Technology Laboratories, Nippon Steel \& Sumitomo Metal Corporation, Amagasaki, Japan. \\ 2. Max-Planck-Institut für Eisenforschung GmbH, Düsseldorf, Germany. \\ 3. Advanced Technology Laboratories, Nippon Steel \& Sumitomo Metal Corporation, Futtsu, Japan.
}

It is nowadays well understood that single crystalline metals exhibit a size dependence of the yield stress: with decreasing pillar diameters an increase in the flow stress is measured in micropillar compression tests. This size effect ("smaller is stronger") is governed by the size of the activated dislocation sources and can be described by a power law equation relating stress and pillar diameter $(\sigma \sim$ $\left.\mathrm{d}^{-\mathrm{n}}\right)$. For single crystalline metallic pillars containing internal particles three different stress - size dependencies are reported in literature: a power law [1, 2], a size-independent (i.e. constant) [3-6], or an intermediate behavior, which can be interpreted as a transition from constant to power law behavior [79]. The differences in size-dependency can be explained by two competing mechanisms, dislocation source activation and dislocation - particle interaction $[8,9]$. When the stress to activate dislocation sources exceeds the particle strengthening effect, pillars should show the "classical" power law dependence. In the present study we want to elaborate if we can quantitatively deduce the particle strengthening effect of e.g. shearable weak or non-shearable strong particles in metal materials from their transition regimes of size dependency. We have examined this idea in precipitation hardened steel with nanometer-sized titanium carbides (TiC) precipitates.

The precipitation hardened steel has a composition of Fe-0.05C-0.50Mn-0.1Ti-3.0Al-0.0009N in mass\%. TiC precipitates were formed during an $8 \mathrm{~h}$ annealing procedure at $660{ }^{\circ} \mathrm{C}$. The $\mathrm{TiC}$ distribution and $\mathrm{TiC} / \mathrm{Fe}$ interface coherency was characterized by aberration-corrected scanning transmission electron microscopy (STEM). To evaluate the number density of TiC particles, the thickness of the TEM samples was measured by electron energy loss spectroscopy (EELS) using the log-ratio technique. Subsequently compression-pillars with diameters ranging from $\sim 100 \mathrm{~nm}$ to $\sim 2300 \mathrm{~nm}$ were fabricated by focused ion beam (FIB) annular milling using $\mathrm{Ga}^{+}$ions. All pillars were oriented with their normal along a $\langle 235\rangle$ direction to facilitate single slip. Two indenter systems were used for compression testing; an ASMEC UNAT II installed inside a scanning electron microscope (SEM) was employed for larger pillars, while in-situ transmission electron microscope (TEM) compression tests were performed with a Hysitron PI95 stage for smaller pillars. All tests were conducted with a flat-tip diamond punch in displacement controlled mode with a nominal strain rate of $1 \times 10^{-3} \mathrm{~s}^{-1}$. Stress-strain curves were calculated from the load-displacement data by considering the area of the pillar top surfaces and the pillar heights. $0.2 \%$ proof stresses versus pillar diameter were plotted to study the size-scaling regimes.

Low angle annular dark field (LAADF) -STEM imaging revealed three TiC platelet variants all aligned with their habit plane along a $<100>$ direction of the ferrite matrix (Figure 1). The TiC platelets possess the Baker-Nutting orientation relationship with the ferrite matrix. Most TiC platelets reveal a coherent interface at the large platelet faces. The TiC density, size and interparticle spacing distributions were determined to a number density of $1.7 \times 10^{22} \mathrm{~m}^{-3}$, an equivalent volume diameter of $7.3 \pm 3.0 \mathrm{~nm}$, and a mean particle spacing in the slip plane of $107 \pm 29 \mathrm{~nm}$, respectively. An example of the in-situ TEM compression test is presented in Figure 2. The conical pillar (top surface diameter $279 \mathrm{~nm}$ ) deformed first close to the pillar surface as a consequence of the stress concentration. The stress-strain curve shows several strain bursts. Taking all size-dependent measurements into account a transition in the size 
dependency of the $0.2 \%$ proof stress was seen at around $400 \mathrm{~nm}$.

In future studies, this behavior will be compared to semicoherent $\mathrm{TiC}$ particles in the same steel with variations in $\mathrm{TiC}$ particle size and particle spacing.

\section{References:}

[1] R Gu and AHW Ngan, Scripta Materialia 68 (2013), 861.

[2] ZH Aitken, H Fan, JA El-Awady and JR Greer, J. Mechanics and Physics of Solids 76 (2015), 208

[3] CP Frick, S Orso and E Arzt, Acta Materialia 55 (2007), 3845

[4] BG Clark, DS Gianola, O Kraft and CP Frick, Advanced Engineering Materials 12 (2010), 808

[5] B Girault, AS Schneider, CP Frick and E Arzt, Advanced Engineering Materials 12 (2010), 385

[6] C Shin, S Lim, H Jin, P Hosemann and J Kwon, Journal of Nuclear Materials 444 (2014), 43

[7] DM Dimiduk, etal. Modelling and Simulation in Materials Science and Engineering 15 (2007), 135

[8] D Kiener, P Hosemann, SA Maloy and AM Minor, Nature Materials 10 (2011), 608

[9] W Chen, J Zhang, S Cao, Y Pan, M Huang, Q Hu, Q Sun, L Xiao, J Sun, Acta Mater. 117 (2016), 68

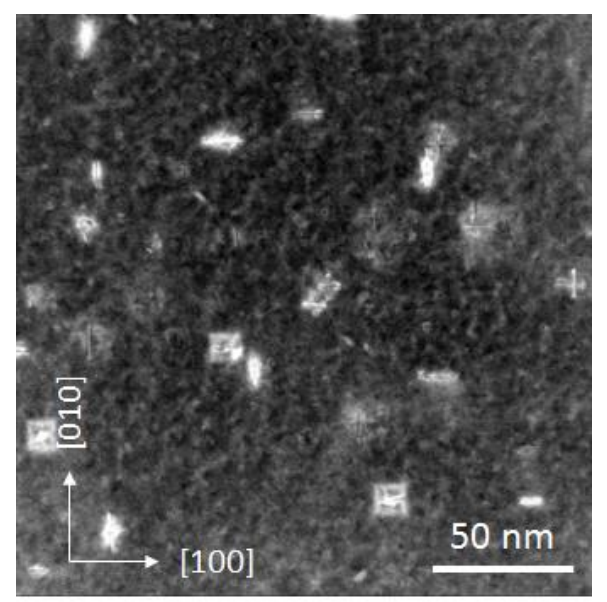

Figure 1. LAADF-STEM image of TiC platelets in a ferrite matrix. The TiC platelets occur as three variants all showing the Baker-Nutting orientation relationship and strain contrast.
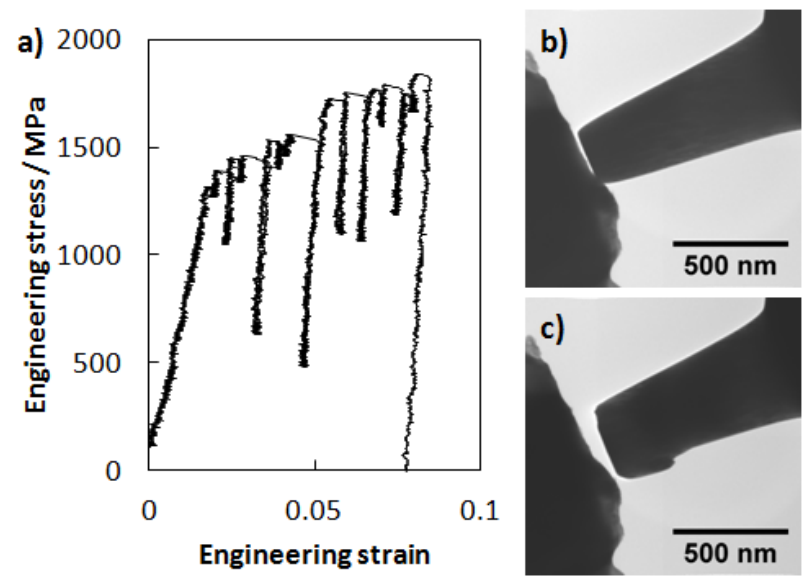

Figure 2. In-situ TEM compression tests. a) Mechanical data, and (b,c) bright field TEM images before and after the compression test. Note the deformation at the pillar top surface. 